\title{
Embeddability and quasi-isometric classification of partially commutative groups
}

\author{
MONTSERRAT CASALS-RuIZ
}

\begin{abstract}
The main goal of this note is to suggest an algebraic approach to the quasi-isometric classification of partially commutative groups (alias right-angled Artin groups). More precisely, we conjecture that if the partially commutative groups $\mathbb{G}(\Delta)$ and $\mathbb{G}(\Gamma)$ are quasi-isometric, then $\mathbb{G}(\Delta)$ is a (nice) subgroup of $\mathbb{G}(\Gamma)$ and vice-versa. We show that the conjecture holds for all known cases of quasi-isometric classification of partially commutative groups, namely for the classes of $n$-trees and atomic graphs. As in the classical Mostow rigidity theory for irreducible lattices, we relate the quasi-isometric rigidity of the class of atomic partially commutative groups with the algebraic rigidity, that is, with the co-Hopfian property of their $\mathbb{Q}$-completions.
\end{abstract}

20A15, 20F36, 20F65, 20F69

\section{Introduction}

A finitely generated group can be considered as a geometric object when endowed with a word metric. Gromov observed that this metric is, in fact, unique up to quasi-isometry and suggested the study of the rigidity problem, that is, when a finitely generated group is quasi-isometric to a given one, and of the classification problem, ie when two groups (in a given class) are quasi-isometric.

In this paper, we focus on the question of quasi-isometric classification of partially commutative groups (also known as right-angled Artin groups) and its connection with the embeddability problem. Recall that a partially commutative group (or a pc group, for short) is a finitely presented group $\mathbb{G}(\Gamma)$ which can be described by a finite simplicial graph $\Gamma$, the commutation graph, in the following way: the vertices of $\Gamma$ are in bijective correspondence with the generators of $\mathbb{G}(\Gamma)$, and the set of defining relations of $\mathbb{G}(\Gamma)$ consists of commutation relations, one for each pair of generators connected by an edge in $\Gamma$.

The quasi-isometric classification of pc groups has been previously considered by Behrstock, Januszkiewic and Neumann, by Bestvina, Kleiner and Sageev, and, more recently, by Huang. Although the results on classification are partial, they already 
exhibit a complex behaviour: on the one hand, some quasi-isometry classes contain many pc groups, while others exhibit some type of rigidity. Furthermore, the techniques used to approach the problem in these cases do not seem to be amenable to address the general classification problem.

The main goal of this note is to suggest an algebraic approach towards the quasiisometric classification of pc groups. More precisely, we study (a stronger version of) the following question.

Question 1 If the pc groups $\mathbb{G}(\Gamma)$ and $\mathbb{G}(\Delta)$ are quasi-isometric, does this imply that $\mathbb{G}(\Gamma)<\mathbb{G}(\Delta)$ and $\mathbb{G}(\Delta)<\mathbb{G}(\Gamma)$ ?

Note that Question 1 has a positive answer if one strengthens the relation to commensurability. That is, if $\mathbb{G}(\Delta)$ and $\mathbb{G}(\Gamma)$ are commensurable, then $\mathbb{G}(\Delta)<\mathbb{G}(\Gamma)$ and $\mathbb{G}(\Gamma)<\mathbb{G}(\Delta)$; see Lemma 26. Although this observation makes Question 1 natural, we will refine the statement and require, not only embeddability between the groups, but rather "nice" embeddability in terms of the extension graphs. The extension graph $\Gamma^{e}$ of a graph $\Gamma$ was introduced in [17] to study the Embeddability problem for pc groups, that is, to determine when a pc group is a subgroup of another one. It is defined as follows: vertices of $\Gamma^{e}$ are in one-to-one correspondence with conjugates of generators of $\mathbb{G}(\Gamma)$, that is,

$$
V\left(\Gamma^{e}\right)=\left\{g^{-1} x g \in \mathbb{G}(\Gamma) \mid x \in V(\Gamma), g \in \mathbb{G}(\Gamma)\right\},
$$

and there is an edge in $\Gamma^{e}$ whenever the elements associated to the corresponding vertices commute in the group, ie

$$
E\left(\Gamma^{e}\right)=\{(u, v) \mid[u, v]=1 \text { in } \mathbb{G}(\Gamma)\} .
$$

In [17], it is shown that if $\Delta$ is an induced subgraph of the extension graph $\Gamma^{e}$, then $\mathbb{G}(\Delta)$ embeds in $\mathbb{G}(\Gamma)$ and that, under some additional conditions on the graphs, the converse also holds. These results suggested that the extension graph could be the graph-theoretical tool to determine when a pc group is a subgroup of another one. However, we show in [10] that, in general, the extension graph is not enough to characterise embeddability: there are pc groups $\mathbb{G}(\Delta)$ and $\mathbb{G}(\Gamma)$ for which there exists an embedding from $\mathbb{G}(\Delta)$ to $\mathbb{G}(\Gamma)$, but $\Delta$ is not an induced subgraph of $\Gamma^{e}$. However, we believe that the extension graph may be helpful for the quasi-isometric classification of pc groups. More formally, we suggest the following conjecture.

Conjecture 2 Let $\Delta$ and $\Gamma$ be simplicial graphs. If $\mathbb{G}(\Delta)$ and $\mathbb{G}(\Gamma)$ are quasiisometric, then $\Delta<\Gamma^{e}$ and $\Gamma<\Delta^{e}$. 
If Conjecture 2 holds, then we would get some algebraic control on the quasi-isometry classes of pc groups. For instance, it would follow that if $\mathbb{G}(\Gamma)$ is a coherent pc group and $\mathbb{G}(\Delta)$ is a pc group quasi-isometric to $\mathbb{G}(\Gamma)$, then $\mathbb{G}(\Delta)$ is also coherent; see Remark 28. Recall that a group is coherent if all its finitely generated subgroups are finitely presentable. Furthermore, we would also gain an algorithmic understanding of the quasi-isometry classes of pc groups. Indeed, it was proven in [8] that there is an algorithm that, given two finite simplicial graphs $\Delta$ and $\Gamma$, decides whether or not $\Delta$ is an induced subgraph of the extension graph $\Gamma^{e}$.

In this note, we show that Conjecture 2 holds for all known cases of quasi-isometric classification of pc groups. The two essential cases that need to be analysed are $n$-trees and atomic graphs.

In [5], the authors studied the problem of classification of graph manifolds and proved that a pc group $\mathbb{G}(\Delta)$ is quasi-isometric to $\mathbb{G}(\Gamma)$, where $\Gamma$ is a tree of diameter greater than 2 , if and only if $\Delta$ is a tree of diameter greater than 2 .

This result was further generalised by Behrstock, Januszkiewic and Neumann for the class of $n$-trees (see Definition 9). The authors prove that, given two $n$-trees $\Delta$ and $\Gamma$, the corresponding pc groups $\mathbb{G}(\Delta)$ and $\mathbb{G}(\Gamma)$ are quasi-isometric if and only if the underlying trees associated to $\Delta$ and $\Gamma$ satisfy a graph-theoretic relation, namely they are bisimilar (see Definition 12).

In a different direction, Bestvina, Kleiner and Sageev (see [7]) introduced and studied the problem of quasi-isometric classification of pc groups for atomic graphs, that is, connected graphs with no valence 1 vertices, no cycles of length less than 5 and no separating closed stars of vertices. They prove that the class of pc groups defined by atomic graphs is quasi-isometrically rigid; that is, given two atomic graphs $\Delta$ and $\Gamma$, the corresponding pc groups $\mathbb{G}(\Delta)$ and $\mathbb{G}(\Gamma)$ are quasi-isometric if and only if $\Delta$ and $\Gamma$ are isomorphic.

Our goal is to prove that Conjecture 2 holds in the aforementioned cases.

Theorem 3 Let $\mathcal{C}$ be one of the following classes of graphs:

- Triangle-built (ie graphs with no induced squares and no induced paths of length more than 2).

- Atomic graphs.

- $n$-trees.

Let $\Delta, \Gamma \in \mathcal{C}$. Then $\mathbb{G}(\Delta)$ is quasi-isometric to $\mathbb{G}(\Gamma)$ if and only if $\Delta<\Gamma^{e}$ and $\Gamma<\Delta^{e}$. 
Remark 4 When this note was already written, Jingyin Huang published a new preprint [15] where he describes pc groups quasi-isometric to pc groups with finite outer automorphism group (a class that naturally extends atomic pc groups). More precisely, Huang shows that if $\mathbb{G}(\Gamma)$ is a pc group with finite outer automorphism group, then $\mathbb{G}(\Delta)$ is quasi-isometric to $\mathbb{G}(\Gamma)$ if and only if $\mathbb{G}(\Gamma)$ and $\mathbb{G}(\Delta)$ are commensurable, if and only if $\Gamma^{e}$ and $\Delta^{e}$ are isomorphic. As a consequence, Conjecture 2 also holds for pc groups with finite outer automorphism group.

In many cases, there is a close relation between the group being quasi-isometrically and algebraically rigid, that is, being co-Hopfian. For instance, in the classical case of irreducible lattices in semisimple Lie groups, this relation is a consequence of Mostow rigidity.

As we mentioned above, the class of atomic pc groups is, in some sense, quasiisometrically rigid, so one can ask how far these groups are from being co-Hopfian. Recall that a group is called co-Hopfian if every injective endomorphism is an automorphism. In this direction, we study the set of injective endomorphisms for the class of atomic pc groups and show that, "up to taking roots," they are automorphisms. More precisely, we prove the following.

Corollary 5 Let $\Gamma$ be an atomic graph and $\psi: \mathbb{G}(\Gamma) \rightarrow \mathbb{G}(\Gamma)$ an injective endomorphism. Then there exist $g \in \mathbb{G}(\Gamma), \sigma \in \operatorname{Aut}(\Gamma)$ and $k_{v} \in \mathbb{Z} \backslash 0, v \in V(\Gamma)$, such that

$$
\psi(v)=g^{-1} \sigma(v)^{k_{v}} g, \quad v \in V(\Gamma) .
$$

In other words, up to conjugacy, graph automorphism and taking powers, $\psi$ is the identity endomorphism.

In the spirit of classical theorems for abelian and locally nilpotent groups, we show in [9] that every pc group embeds into a divisible group, its $\mathbb{Q}$-completion, which, roughly speaking, is the smallest divisible group containing $\mathbb{G}(\Gamma)$. In other words, the $\mathbb{Q}$-completion of $\mathbb{G}(\Gamma)$ is an initial object in the category of divisible $\mathbb{G}(\Gamma)$-groups (ie divisible groups with a designated copy of $\mathbb{G}(\Gamma)$ ). From the description of the set of injective endomorphisms of an atomic pc group, one deduces that, although atomic pc groups are not co-Hopfian, their $\mathbb{Q}$-completions are.

Corollary 6 Let $\Gamma$ be an atomic graph. Then the $\mathbb{Q}$-completion $\mathbb{G}(\Gamma)^{\mathbb{Q}}$ of $\mathbb{G}(\Gamma)$ is co-Hopfian.

In this context, it is natural to ask if the correspondence between quasi-isometric and algebraic rigidities holds in general for the class of pc groups. More precisely, we 
call a pc group $\mathbb{G}(\Delta)$ weakly quasi-isometrically rigid if its quasi-isometry class is determined by the isomorphism type of its extension graph, that is, $\mathbb{G}(\Delta)$ is quasiisometric to $\mathbb{G}(\Gamma)$ if and only if the extension graph $\Delta^{e}$ is isomorphic to $\Gamma^{e}$. In this terminology, we ask the following.

Question 7 Is it true that $\mathbb{G}(\Gamma)$ is weakly quasi-isometrically rigid if and only if the $\mathbb{Q}$-completion $\mathbb{G}(\Gamma)^{\mathbb{Q}}$ of $\mathbb{G}(\Gamma)$ is co-Hopfian?

We assume that the reader is familiar with basics of the theory of partially commutative groups. We refer the reader to [8] and references there for preliminaries and notation.

\section{Elementary cases}

In this section we review some cases of quasi-isometric classification of pc groups, namely three classical cases: free groups, free abelian groups and direct products of two free groups; and the case of pc groups defined by trees and triangle-built graphs.

In the case of free and free abelian groups, we have a complete classification: a finitely generated group $G$ is quasi-isometric to the free abelian group $\mathbb{Z}^{n}$ if and only if it is virtually $\mathbb{Z}^{n}$ (see [14]), and if $G$ is quasi-isometric to a (nonabelian) free group, then it is commensurable to it (and $G$ acts geometrically on a tree). When we restrict our consideration to the class of pc groups, we deduce that a pc group $\mathbb{G}(\Delta)$ is quasiisometric to $\mathbb{Z}^{n}$ if and only if $\mathbb{G}(\Delta)$ is isomorphic to $\mathbb{Z}^{n}$, and a pc group $\mathbb{G}(\Delta)$ is quasi-isometric to a nonabelian free group $F_{n}$ if and only if $\mathbb{G}(\Delta)$ is a nonabelian free group $F_{m}$.

In the free abelian case, Conjecture 2 holds trivially. Furthermore, the converse also holds. Indeed, the graph associated to $\mathbb{Z}^{n}$ is a clique $\Gamma$ of dimension $n$. Hence, if $\Delta$ is an induced subgraph of the extension graph $\Gamma^{e}=\Gamma$, then $\Delta$ is a clique of dimension less than or equal to $n$. Furthermore, if $\Gamma<\Delta^{e}=\Delta$, then it follows that $\Delta=\Gamma$.

If $\mathbb{G}(\Gamma)$ is a nonabelian free group, then the extension graph associated to $\Gamma$ is an infinite edgeless graph, and so Conjecture 2 holds. In this case, the converse is also true: if $\Delta<\Gamma^{e}$, then the graph of $\Delta$ is edgeless and so $\mathbb{G}(\Delta)$ is a free group. If $\Gamma<\Delta^{e}$, it follows that $\Delta$ has at least two vertices and so $\mathbb{G}(\Delta)$ is a nonabelian free group.

These results were generalised to groups acting on direct products of trees by several authors (see $[16 ; 20 ; 1]$ ): if a group $G$ is quasi-isometric to the direct product of two nonabelian free groups $F_{n} \times F_{m}$, then $G$ acts geometrically on the direct product of two trees. In particular, a pc group $\mathbb{G}(\Delta)$ is quasi-isometric to $F_{n} \times F_{m}$ if and only if $\mathbb{G}(\Delta)$ is the direct product of two nonabelian free groups $F_{r} \times F_{S}$. 
The extension graph $\Gamma^{e}$ associated to $F_{k} \times F_{l}$ is the join graph of two infinite edgeless graphs, and so it is immediate to check that Conjecture 2 holds. The converse also holds: any induced subgraph $\Delta$ of $\Gamma^{e}$ is either edgeless or a join. If $\Gamma<\Delta^{e}$, it follows that $\Delta^{e}$ is the join of two infinite edgeless graphs, and so $\mathbb{G}(\Delta)$ is isomorphic to $F_{k^{\prime}} \times F_{l^{\prime}}$ for $k^{\prime}, l^{\prime}>1$.

We now turn our attention to the class of pc groups whose finitely generated subgroups are pc groups. In [13], Droms gives a graph-theoretic characterisation of this class: every finitely generated subgroup of $\mathbb{G}(\Gamma)$ is a pc group if and only if $\Gamma$ is triangle-built, that is, $\Gamma$ contains no induced squares and no induced paths of diameter more than 2 . In this case, Droms shows that $\mathbb{G}(\Gamma)$ is isomorphic to $\mathbb{Z}^{n} \times \mathbb{G}\left(\Gamma^{\prime}\right)$, where $\Gamma^{\prime}$ is the disjoint union of triangle-built graphs or, equivalently, $\mathbb{G}\left(\Gamma^{\prime}\right)$ is the free product of triangle-built pc groups. By [16], we have that $\mathbb{G}(\Delta)$ is quasi-isometric to $\mathbb{G}(\Gamma)$ if and only if $\mathbb{G}(\Delta)$ is isomorphic to $\mathbb{Z}^{n} \times \mathbb{G}\left(\Delta^{\prime}\right)$ and $\mathbb{G}\left(\Gamma^{\prime}\right)$ and $\mathbb{G}\left(\Delta^{\prime}\right)$ are quasi-isometric. Then, by [22] it follows that each (one-ended) factor in the Grushko decomposition of $\mathbb{G}\left(\Gamma^{\prime}\right)$ is equivalent to a (one-ended) factor in the Grushko decomposition of $\mathbb{G}(\Delta)$ and vice-versa. By induction on the number of vertices in $\mathbb{G}\left(\Gamma^{\prime}\right)$, we conclude that $\Delta^{\prime}<\left(\Gamma^{\prime}\right)^{e}$ and $\Gamma^{\prime}<\left(\Delta^{\prime}\right)^{e}$, and so Conjecture 2 also holds in this case.

Let $T$ be a tree of diameter 2 , ie $\mathbb{G}(T)$ is isomorphic to $\mathbb{Z} \times F_{k}$ for $k>1$. Then $\mathbb{G}(\Delta)$ is quasi-isometric to $\mathbb{G}(T)$ if and only if $\mathbb{G}(\Delta)$ is isomorphic to $\mathbb{Z} \times F_{n}$ for $n>1$. On the other hand, $\Delta<T^{e}$ and $T<\Delta^{e}$ if and only if $\mathbb{G}(\Delta)$ is isomorphic to $\mathbb{Z} \times F_{n}$ for $n>1$.

The quasi-isometric classification for trees of diameter greater than or equal to 3 was established by Behrstock and Neumann.

Theorem 8 [5, Theorem 5.3] Let $T$ be a tree of diameter greater than or equal to 3. Then $\mathbb{G}(\Gamma)$ is quasi-isometric to $\mathbb{G}(T)$ if and only if $\Gamma$ is a tree of diameter greater than or equal to 3 .

It is easy to see (for instance, see [17]) that any tree is an induced subgraph of the extension graph $T^{e}$ of a tree $T$ of diameter greater than or equal to 3 .

On the other hand, since the extension graph of a tree is a tree, if $\Gamma$ is connected, it follows that $\Gamma<T^{e}$ if and only if $\Gamma$ is a tree. If $T<\Gamma^{e}$, then we have that the diameter of $\Gamma$ is greater than or equal to 3 . In this case, we have shown that $\mathbb{G}(\Gamma)$ is quasi-isometric to $\mathbb{G}(T)$, where $T$ is a tree if and only if $\Gamma<T^{e}, T<\Gamma^{e}$, and $\Gamma$ is connected. 


\section{3 n-trees}

The class of $n$-trees was introduced and studied by Behrstock, Januszkiewic and Neumann in [4]. We next recall some basic definitions and results and refer the reader to [4] for further details.

Definition 9 We define the class of $n$-trees $\mathcal{T}_{n}$ to be the smallest class of $n$-dimensional simplicial complexes satisfying:

- The $n$-simplex is in $\mathcal{T}_{n}$.

- If $K_{1}$ and $K_{2}$ are complexes in $\mathcal{T}_{n}$, then the union of $K_{1}$ and $K_{2}$ along any $(n-1)$-simplex is in $\mathcal{T}_{n}$.

For $n=1$ this is the class of finite trees. In this section, we consider pc groups defined by $n$-trees. Although, formally, we should define these pc groups by the 1 -skeleton of the $n$-tree, we abuse the notation and write $\mathbb{G}(K)$ where $K \in \mathcal{T}$.

Fix a complex $K \in \mathcal{T}_{n}$. We define a piece to be the star in $K$ of an $(n-1)$-simplex of $K$ which is the boundary of at least two $n$-simplices. Let $P$ denote a piece of $K$. Then $P$ consists of a finite collection of $n$-simplices attached along the common $(n-1)$-simplex, ie the join of the $(n-1)$-simplex with a finite set of points $p_{1}, \ldots, p_{k}$.

Definition 10 To each $K \in \mathcal{T}_{n}$, we associate a labelled bipartite tree $\operatorname{gph}(K)$ as follows. To each piece in $K$ we assign a vertex labelled $p$ (for piece). To each of the $n$-simplices that is in more than one piece, we assign a vertex labelled $f$ (for face). Each $f$-vertex is connected by an edge to each of the $p$-vertices that corresponds to a piece containing the $n$-simplex.

For any $K \in \mathcal{T}_{n}$, since there is a simplicial map to an $n$-dimensional simplex $\Delta$, which is unique up to permutation of $\Delta$, it follows that a labelling on the vertices of $\Delta$ by 1 to $n+1$ pulls back to a consistent labelling on all the vertices of $K$. Note that, in any piece, all the vertices of their common $(n-1)$-simplex (the "spine" of the piece) are given the same label. We label each $p$-vertex by the index of the $n$-simplex vertex which is not on the spine of the corresponding piece. Hence, the label set for the $p$-vertices are the elements of the set $\{1, \ldots, n+1\}$. The possible labels for vertices are thus $p 1, p 2, \ldots, p(n+1)$ and $f$, for a total of $n+2$ possible labels. The $p / f$-distinction gives a bipartite structure on our tree $\operatorname{gph}(K)$. The $p$-vertices to which a given $f$-vertex is connected have distinct labels, so an $f$-vertex has valence at most $n+1$ (and at least 2). A $p$-vertex can be connected to any number of $f$-vertices. 
Note that the graph $\operatorname{gph}(K)$ associated to an $n$-tree corresponds to the graph of the graph-of-groups decomposition of $\mathbb{G}(K)$ where vertex groups are fundamental groups of the pieces and edge groups are labelled by the fundamental group of an $n$-simplex (ie edge groups are free abelian).

Definition 11 A coloured graph is a graph $\Gamma$, a set $C$, and a "vertex colouring" $c: V(\Gamma) \rightarrow C$. A weak covering of coloured graphs is a graph homomorphism $f: \Gamma \rightarrow$ $\Gamma^{\prime}$ which respects colours and has the following property: for each $v \in V(\Gamma)$ and for each edge $e^{\prime} \in E\left(\Gamma^{\prime}\right)$ at $f(v)$, there exists an $e \in E(\Gamma)$ at $v$ with $f(e)=e^{\prime}$.

Henceforth, we assume that all graphs under consideration are connected. It is easy to see that a weak covering is then surjective.

Definition 12 Coloured graphs $\Gamma_{1}$ and $\Gamma_{2}$ are bisimilar if $\Gamma_{1}$ and $\Gamma_{2}$ weakly cover some common coloured graph.

The main result of [4] is the quasi-isometric classification of $n$-trees in terms of bisimilarity of the defining graphs. More precisely, the authors prove:

Theorem 13 [4, Theorem 1.1] Let $K, K^{\prime} \in \mathcal{T}_{n}$. The groups $\mathbb{G}(K)$ and $\mathbb{G}\left(K^{\prime}\right)$ are quasi-isometric if and only if $\operatorname{gph}(K)$ and $\operatorname{gph}\left(K^{\prime}\right)$ are bisimilar after possibly reordering the $p$-colours by an element of the symmetric group on $n+1$ elements.

The goal of this section is to show that the graphs associated to $n$-trees $\Delta$ and $\Gamma$ are bisimilar (after possibly reordering the $p$-colours) if and only if $\Delta<\Gamma^{e}$ and $\Gamma<\Delta^{e}$.

Before we turn our attention to the proof, we recall an easy but very useful lemma that describes the extension graph $\Gamma^{e}$ as a sequence of "doublings" over stars of vertices.

Lemma 14 [17, Lemma 22] Let $\Gamma$ be a finite graph and $\Delta$ be a finite induced subgraph of $\Gamma^{e}$. Then there exists an $l>0$, a sequence of vertices $v_{1}, v_{2}, \ldots, v_{l}$ of $\Gamma^{e}$, and a sequence of finite induced subgraphs $\Gamma=\Gamma_{0} \leq \Gamma_{1} \leq \cdots \leq \Gamma_{l}$ of $\Gamma^{e}$, where $\Gamma_{i}$ is obtained by taking the double of $\Gamma_{i-1}$ along $\operatorname{Star}_{\Gamma_{i-1}}\left(v_{i}\right)$ for each $i=1, \ldots, l$, such that $\Delta \leq \Gamma_{l}$.

Lemma 15 Assume that $\operatorname{gph}(\Delta)$ weakly covers $\operatorname{gph}(\Gamma)$; then $\Delta<\Gamma^{e}$.

Proof Assume that $f: \operatorname{gph}(\Delta) \rightarrow \operatorname{gph}(\Gamma)$ is a weak covering. Since both graphs are connected and without multiple edges, $f$ is surjective, and so the graph $\operatorname{gph}(\Gamma)$ is an induced subgraph of $\operatorname{gph}(\Delta)$. 
We prove the statement by induction on the number $m=|V(\operatorname{gph}(\Delta)) \backslash V(\operatorname{gph}(\Gamma))|$.

Induction base Let $m=0$. In this case, the $\operatorname{gph}(\Gamma)$ is an induced subgraph with the same set of vertices, and so it coincides with $\operatorname{gph}(\Delta)$. It follows from the definition of $\operatorname{gph}(\Delta)$ and $\operatorname{gph}(\Gamma)$ that $\Delta$ and $\Gamma$ only differ by their "leaves"; that is, they differ by the set of $n$-simplices such that only one of the $(n-1)$-faces of each is a face of another $n$-simplex.

Define the core of an $n$-tree $\Lambda$ to be the $n$-subtree of $\Lambda$ that consists of $n$-simplices that are not leaves of $\Lambda$. In this terminology, if $m=0$, then the cores of $\Delta$ and $\Gamma$ are isomorphic (possibly empty). We denote by $\varphi^{\prime}$ a colour preserving isomorphism from the core of $\Delta$ to the core of $\Gamma$.

Our goal is to define an embedding $\varphi$ from $\Delta$ to $\Gamma^{e}$ that extends the isomorphism $\varphi^{\prime}$. Without loss of generality, assume that $\Delta$ and $\Gamma$ are different; that is, there exists an $(n-1)$-simplex $F$ that is a face of at least two $n$-simplices where one of these simplices is a leaf, and the pieces associated to $F$ in $\Delta$ and in $\Gamma$ differ in the number of leaves. For the $n$-simplices that contain the face $F$ in $\Gamma$ (resp. in $\Delta$ ) and which belong to the core, we denote by $x_{1}, \ldots, x_{r}$ (resp. $\left.x_{1}^{\prime}, \ldots, x_{r}^{\prime}\right)$ the vertices of these simplices that do not belong to the face $F$ in $\Gamma$ (resp. in $\Delta$ ). For the other $n$-simplices, that is, the leaves that contain $F$, we denote by $y_{1}, \ldots, y_{k}$ (resp. $\left.y_{1}^{\prime}, \ldots, y_{l}^{\prime}, l>k>0\right)$ the vertices that do not belong to the face $F$ in $\Gamma$ (resp. in $\Delta$ ). Note that $r$ may be 0 , in which case $k \geq 2$, and $\operatorname{gph}(\Gamma)$ is a vertex.

We define an embedding from the core of $\Delta$, together with the piece associated to $F$, to $\Gamma^{e}$ as follows: $\varphi$ on the core of $\Delta$ is defined as $\varphi^{\prime}$ (and so, in particular, $\varphi\left(x_{i}^{\prime}\right)=x_{i}$ for $i=1, \ldots, r)$, and

$$
\varphi\left(y_{i}^{\prime}\right)= \begin{cases}y_{1}^{\left(x_{1}^{i}\right)} & \text { for } i=1, \ldots, l \text { if } r \neq 0, \\ y_{1}^{\left(y_{2}^{i}\right)} & \text { otherwise. }\end{cases}
$$

It is easy to check that the map $\varphi$ induces a graph embedding, since $y_{i}$ and $x_{j}$ do not commute with each other for $i=1, \ldots, k$ and $j=1, \ldots, r$, but they commute with the vertices in the face $F$.

Repeating the above argument for the pieces for which the number of leaves in $\Delta$ is different than that of $\Gamma$, one obtains an embedding from $\Delta$ to $\Gamma^{e}$. Note that, by construction, vertices of a piece $P$ (resp. face) in $\Delta$ that is identified to a vertex $v$ in $\operatorname{gph}(\Delta)$ are sent to conjugates of vertices of the piece $P^{\prime}$ (resp. face) in $\Gamma$ that is identified to the same vertex $v$ in $\operatorname{gph}(\Gamma)$. Furthermore, vertices of faces in $\Delta$ that are identified with vertices of $\operatorname{gph}(\Delta)$ are sent to the same conjugate (in this case, the conjugating element is trivial). 
Induction step Let $v$ be a leaf of $\operatorname{gph}(\Delta)$ such that the weak covering $f$ restricted to $\operatorname{gph}(\Delta) \backslash\{v\}$ is again a weak covering of graphs. Note that such a $v$ exists because $\operatorname{gph}(\Delta)$ and $\operatorname{gph}(\Gamma)$ are trees and $|V(\Delta)| \ngtr|V(\Gamma)|$. Assume by induction that there is an embedding $\psi$ from the $n$-tree $\Delta^{\prime}$ associated to $\operatorname{gph}(\Delta) \backslash\{v\}$ into $\Gamma^{e}$ such that

- vertices of a piece $P$ in $\Delta^{\prime}$ that is identified to a $p$-vertex $v$ in $\operatorname{gph}\left(\Delta^{\prime}\right)$ are sent to conjugates of vertices of the piece $P^{\prime}$ in $\Gamma$ that is identified to the vertex $f(v)$ in $\operatorname{gph}(\Gamma)$, and

- vertices of a face $F$ in $\Delta^{\prime}$ that is identified to an $f$-vertex $w$ in $\operatorname{gph}\left(\Delta^{\prime}\right)$ are sent to the same conjugate of vertices of the face $F^{\prime}$ in $\Gamma$ that is identified to the $f$-vertex $f(w)$ in $\operatorname{gph}(\Gamma)$.

By Lemma 14, $\Delta^{\prime}$ embeds into $\Gamma_{n-1}<\Gamma^{e}$, where $\Gamma_{n-1}$ is obtained from $\Gamma$ by a sequence of doublings.

Let $w$ be the vertex of $\operatorname{gph}(\Delta)$ so that $(v, w) \in E(\operatorname{gph}(\Delta))$. Note that, from the construction of $\operatorname{gph}(\Delta)$, it follows that $v$ is a $p$-vertex and $w$ is an $f$-vertex. By induction, the embedding of $\Delta^{\prime}$ into $\Gamma_{n-1}$ satisfies that the image under $\psi$ of vertices $a_{i}$ in $\Delta$ associated to the face identified with $w$ is $b_{j_{i}}^{g_{n-1}}$, where $g_{n-1} \in \mathbb{G}(\Gamma)$ and $b_{j_{i}}$ is a vertex in the face identified with $f(w)$. Let $h_{n} \in \mathbb{G}(\Gamma)$ be so that the alphabet of $h_{n}$ is exactly the alphabet that labels the face $F^{\prime}$ identified with $f(w)$. We can choose $h_{n}$ so that the element $g_{n}=h_{n}^{g_{n-1}^{-1}}$ has not appeared in the (finite) sequence of doubling used to construct $\Gamma_{n-1}$. Indeed, there are at most $n-1$ doublings, and the subgroup associated to faces are free abelian groups of rank at least 2 . Conjugating by $g_{n}$, we obtain a doubling of $\Gamma_{n-1}$ along $F^{\prime g_{n-1}}=F^{g_{n-1} g_{n}}$; that is, $\Gamma_{n}=\Gamma_{n-1} \sqcup_{F^{\prime} g_{n}} \Gamma_{n-1}^{g_{n}}$.

If the piece identified with $v$ in $\operatorname{gph}(\Delta)$ is the same as the piece identified with $f(v)$ in $\Gamma$, then the embedding $\psi$ of $\Delta^{\prime}$ into $\Gamma^{e}$ can be extended to an embedding of $\Delta$. Indeed, it suffices to send the vertices $a_{i}$ of the $n$-simplices of said piece that are not in the face $F$ to $\left(b_{i}^{g_{n-1}}\right)^{g_{n}}$, where $b_{i}$ are the corresponding vertices in the piece in $\Gamma$ that are not in the face identified with $f(w)$.

If the pieces have different numbers of leaves, we define the embedding $\psi$ as in the base of induction; that is, $\psi$ sends the vertices $a_{i}$ of the $n$-simplices of said piece that are not in the face $F$ to $\left(\varphi\left(b_{i}\right)^{g_{n-1}}\right)^{g_{n}}$, where $b_{i}$ are the corresponding vertices in the piece in $\Gamma$ that are not in the face identified with $f(w)$. By construction, the embedding $\psi$ satisfies the induction hypothesis.

Corollary 16 Let $\Gamma, \Delta \in \mathcal{T}_{n}$. If $\operatorname{gph}(\Delta)$ and $\operatorname{gph}(\Gamma)$ are bisimilar, then $\Delta<\Gamma^{e}$ and $\Gamma<\Delta^{e}$. 
Proof If $\operatorname{gph}(\Delta)$ and $\operatorname{gph}(\Gamma)$ are bisimilar, it follows from the definition that they weakly cover a graph $\operatorname{gph}(\Lambda)$. Since the graphs are assumed to be connected, it follows that the weak covering is an epimorphism, and so, in particular, there are embeddings of $\operatorname{gph}(\Lambda)$ into $\operatorname{gph}(\Gamma)$ and into $\operatorname{gph}(\Delta)$. Hence, $\Lambda<\Gamma$ and $\Lambda<\Delta$, and so $\Lambda^{e}<\Gamma^{e}$ and $\Lambda^{e}<\Delta^{e}$. It follows from Lemma 15 that $\Delta<\Lambda^{e}<\Gamma^{e}$ and $\Gamma<\Lambda^{e}<\Delta^{e}$.

Lemma 17 Let $\Gamma, \Delta \in \mathcal{T}_{n}$. Then if $\Delta<\Gamma^{e}$ and $\Gamma<\Delta^{e}$, then $\operatorname{gph}(\Delta)$ and $\operatorname{gph}(\Gamma)$ are bisimilar.

Proof Let us first show that one can assume $\Delta$ to be minimal, that is, for any proper subgraph $\Delta^{\prime}$ of $\Delta$ such that $\Delta^{\prime} \in \mathcal{T}_{n}$, the graph $\operatorname{gph}(\Delta)$ is not bisimilar to $\operatorname{gph}\left(\Delta^{\prime}\right)$. Let $\Delta^{\prime}$ be a subgraph of $\Delta$ such that $\Delta^{\prime} \in \mathcal{T}_{n}$ and the graphs $\operatorname{gph}\left(\Delta^{\prime}\right)$ and $\operatorname{gph}(\Delta)$ are bisimilar. If $\Delta<\Gamma^{e}$ and $\Gamma<\Delta^{e}$, then $\Delta^{\prime}<\Gamma^{e}$ and $\Gamma<\left(\Delta^{\prime}\right)^{e}$. Indeed, $\Delta^{\prime}<\Delta$ and, by assumption, $\Delta<\Gamma^{e}$; hence, $\Delta^{\prime}<\Gamma^{e}$. On the other hand, by assumption, $\Gamma<\Delta^{e}$, and since $\operatorname{gph}(\Delta)$ and $\operatorname{gph}\left(\Delta^{\prime}\right)$ are bisimilar, by Lemma 15 , we have that $\Delta^{e}<\left(\Delta^{\prime}\right)^{e}$, and so $\Gamma<\left(\Delta^{\prime}\right)^{e}$. Furthermore, if the statement holds for $\Delta^{\prime}$ and $\Gamma$, that is, if $\operatorname{gph}\left(\Delta^{\prime}\right)$ and $\operatorname{gph}(\Gamma)$ are bisimilar, then we conclude that the statement also holds for $\Delta$ and $\Gamma$. Since $\operatorname{gph}(\Delta)$ is bisimilar to $\operatorname{gph}\left(\Delta^{\prime}\right)$ and by transitivity of the relation, $\operatorname{gph}(\Delta)$ and $\operatorname{gph}\left(\Delta^{\prime}\right)$ are bisimilar.

We further assume that $\Delta$ is minimal. Observe that if $\Gamma, \Gamma^{\prime}$ and $\Lambda$ are $n$-trees, $\Lambda<\Gamma$ and $\Lambda<\Gamma^{\prime}$, then $\Gamma \sqcup_{\Lambda} \Gamma^{\prime}$ is also an $n$-tree. Furthermore,

$$
\operatorname{gph}\left(\Gamma \sqcup_{\Lambda} \Gamma^{\prime}\right)=\operatorname{gph}(\Gamma) \sqcup_{\operatorname{gph}(\Lambda)} \operatorname{gph}\left(\Gamma^{\prime}\right) .
$$

By Lemma 14, we have that if $\Delta$ is a finite induced subgraph of $\Gamma^{e}$, then there exist $l>0$, a sequence of vertices $v_{1}, v_{2}, \ldots, v_{l}$ of $\Gamma^{e}$, and a sequence of finite induced subgraphs $\Gamma=\Gamma_{0} \leq \Gamma_{1} \leq \cdots \leq \Gamma_{l}$ of $\Gamma^{e}$, where $\Gamma_{i}$ is obtained by taking the double of $\Gamma_{i-1}$ along $\operatorname{Star}_{\Gamma_{i-1}}\left(v_{i}\right)$ for each $i=1, \ldots, l$, such that $\Delta \leq \Gamma_{l}$.

Note that the star of a vertex of an $n$-tree $\Gamma$ is an $n$-tree. If we assume by induction that $\Gamma_{l-1}$ is an $n$-tree (and so is $\operatorname{Star}_{\Gamma_{l-1}}(v)$ for every vertex in $\Gamma_{l-1}$ ), then it follows from the above observation that the double $\Gamma_{l}$ over the $n$-tree $\operatorname{Star}_{\Gamma_{l-1}}\left(v_{l-1}\right)$ is again an $n$-tree. Furthermore, the double of a tree $T$ over a subtree $T^{\prime}$, ie $T \sqcup_{T^{\prime}} T$, is bisimilar to $T$. Thus, by induction, $\operatorname{gph}\left(\Gamma_{l}\right)$ is bisimilar to $\operatorname{gph}(\Gamma)$.

Since $\Delta<\Gamma_{l}$ and $\Delta$ is an $n$-tree, it follows that $\operatorname{gph}(\Delta)$ is a subtree of $\operatorname{gph}\left(\Gamma_{l}\right)$. Since $\operatorname{gph}\left(\Gamma_{l}\right)$ is bisimilar to $\operatorname{gph}(\Gamma)$, it follows that $\operatorname{gph}(\Delta)$ is bisimilar to a subgraph of $\operatorname{gph}(\Gamma)$.

Since $\Gamma<\Delta^{e}$ by assumption, it follows that either $\Gamma<\Delta$ (and so $\operatorname{gph}(\Gamma)<\operatorname{gph}(\Delta)$ ) or the above argument applies, and so $\operatorname{gph}(\Gamma)$ is bisimilar to a subgraph of $\operatorname{gph}(\Delta)$. 
Since $\operatorname{gph}(\Delta)$ is bisimilar to a subgraph of $\operatorname{gph}(\Gamma)$, and $\operatorname{gph}(\Gamma)$ is in turn bisimilar to a subgraph of $\operatorname{gph}(\Delta)$, it follows that $\Delta$ is bisimilar to a subgraph of itself. Since $\Delta$ is minimal (ie not bisimilar to any proper subgraph) by assumption, we conclude that the subgraph is $\Delta$ and so $\Delta<\Gamma$ and $\Gamma$ is bisimilar to $\Delta$.

We summarise the results of this section in the following corollary.

Corollary 18 Let $\Gamma, \Delta \in \mathcal{T}_{n}$. Then $\mathbb{G}(\Gamma)$ and $\mathbb{G}(\Delta)$ are quasi-isometric if and only if $\operatorname{gph}(\Delta)$ and $\operatorname{gph}(\Gamma)$ are bisimilar, if and only if $\Delta<\Gamma^{e}$ and $\Gamma<\Delta^{e}$.

\section{Atomic graphs}

In this section we study the class of atomic pc groups introduced by Bestvina, Kleiner and Sageev in [7]. Recall that an atomic graph is a graph with no valence 1 vertices, no cycles of length less than 5 and no separating closed stars of vertices. More precisely, in this section, we focus on the algebraic rigidity of the class of atomic pc groups and show that, if an atomic pc group $\mathbb{G}(\Delta)$ embeds into an atomic pc group $\mathbb{G}(\Gamma)$ and vice-versa, then the groups $\mathbb{G}(\Delta)$ and $\mathbb{G}(\Gamma)$ are isomorphic.

In order to study the group embeddability into an atomic pc group $\mathbb{G}(\Gamma)$, it suffices to study the graph embeddability into the extension graph $\Gamma^{e}$; see [17]. We begin by recalling the following technical lemma, which follows from the proof of [17, Lemma 26(6)] and summarises the tree-like properties of the extension graph $\Gamma^{e}$.

Lemma 19 (See [17, Lemma 26]) Let $\Gamma$ be an atomic graph, and let $\Gamma^{g}$ and $\Gamma^{h}$ be two subgraphs of the extension graph $\Gamma^{e}$. Then:

(1) The intersection of $\Gamma^{g}$ and $\Gamma^{h}$ is either empty or is contained in the star $\operatorname{Star}_{\Gamma^{e}}(v)$ of some vertex $v \in \Gamma^{g} \cap \Gamma^{h}$, and $\Gamma^{g} \cap \Gamma^{h}=\operatorname{Star}_{\Gamma^{g}}(v)=\operatorname{Star}_{\Gamma^{h}}(v)$.

(2) The star of any vertex disconnects the extension graph $\Gamma^{e}$; moreover, any two vertices $v$ and $w$ that do not belong to the same conjugate of $\Gamma$ in $\Gamma^{e}$ can be separated by the star of some vertex of $\Gamma^{e}$.

(3) If $\Gamma^{g}$ and $\Gamma^{h}$ are nontrivial and $\Gamma^{g} \cap \Gamma^{h}=\operatorname{Star}_{\Gamma^{g}}(v)=\operatorname{Star}_{\Gamma^{h}}(v)$, then $\Gamma^{g} \cap \Gamma^{h}$ disconnects the extension graph $\Gamma^{e}$.

(4) If $p=\left(v_{1}, \ldots, v_{k}, w_{1}, \ldots, w_{l}, v_{k+1}, \ldots, v_{m}\right)$ is a simple path in $\Gamma^{e}$ so that $v_{i} \in \Gamma$ and $w_{j} \in \Gamma^{e} \backslash \Gamma$ for $i=1, \ldots, m$ and $j=1, \ldots, l$, then there exists $u \in \Gamma$ so that $v_{k}, v_{k+1} \in \operatorname{Star}_{\Gamma}(u)$. 
We next show that the embeddability of an atomic graph $\Gamma$ into the corresponding extension graph $\Gamma^{e}$ is rigid; that is, any embedding $\varphi: \Gamma \rightarrow \Gamma^{e}$ is the identity up to an automorphism.

Theorem 20 Let $\Gamma$ be an atomic graph and $\varphi: \Gamma \rightarrow \Gamma^{e}$ an embedding of $\Gamma$ into the extension graph $\Gamma^{e}$ (as a full subgraph). Then there exists $g \in \mathbb{G}(\Gamma)$ and an automorphism $\alpha$ of $\Gamma$ so that $\varphi(\alpha(\Gamma))=\Gamma^{g}$, ie up to conjugacy and graph automorphism there is only one way to embed $\Gamma$ into $\Gamma^{e}$.

Proof Let $\Gamma$ be an atomic graph and let $n$ be the rank of $\pi_{1}(\Gamma)\left(\pi_{1}(\Gamma)\right.$ is isomorphic to the free group $F_{n}$ of rank $n$ ). Recall that, since $\Gamma$ is atomic, there are no vertices of valence one. We mark $n$ cycles in $\Gamma$ as follows. If $n=1$, then $\pi_{1}(\Gamma)$ is isomorphic to $\mathbb{Z}$, and we mark the only cycle in $\Gamma$. Let $T^{\prime}$ be a maximal subtree of $\Gamma$ and $\Gamma \backslash T^{\prime}$ be the set of edges $\left\{e_{1}, \ldots, e_{n}\right\}$. By the length of a cycle $c$, denoted by $|c|$, we mean the length of its core. As usual, if we fix a base point in $T^{\prime}$, each edge $e_{i}$ defines a cycle $c_{T^{\prime}, e_{i}}$ in $\Gamma$ for $i=1, \ldots, n$. Note that, by definition, the length of the cycle defined by an edge $e_{i}$ is independent of the choice of the base point.

Re-enumerating if necessary, we shall assume that $\left|c_{T^{\prime}, e_{i}}\right| \leq\left|c_{T^{\prime}, e_{j}}\right|$ for $1 \leq i<j \leq n$. Every maximal subtree $T^{\prime}$ of $\Gamma$ defines a tuple $\left(\left|c_{T^{\prime}, e_{1}}\right|, \ldots,\left|c_{T^{\prime}, e_{n}}\right|\right)$ (independent of the choice of the base point). The natural lexicographical order on the tuples $\left(\left|c_{T^{\prime}, e_{1}}\right|, \ldots,\left|c_{T^{\prime}, e_{n}}\right|\right)$, induces an order on the set of maximal subtrees of $\Gamma$, namely $T<T^{\prime}$ if $\left(\left|c_{T, e_{1}}\right|, \ldots,\left|c_{T, e_{n}}\right|\right)$ is less than $\left(\left|c_{T^{\prime}, e_{1}}\right|, \ldots,\left|c_{T^{\prime}, e_{n}}\right|\right)$ in the lexicographical order, that is, there is $i \in\{1, \ldots, n\}$ such that $\left|c_{T, e_{k}}\right|=\left|c_{T^{\prime}, e_{k}}\right|$ for $1 \leq k<i$ and $\left|c_{T, e_{i}}\right|<\left|c_{T^{\prime}, e_{i}}\right|$. Let $T$ be a minimal (in the above order) maximal subtree of $\Gamma$, ie $T \leq T^{\prime}$ for all maximal subtrees $T^{\prime}$ of $\Gamma$. We mark the cores of the cycles $c_{e_{1}, T}, \ldots, c_{e_{n}, T}$. Until the end of the proof of this lemma, unless stated otherwise, by a cycle in $\Gamma$ we mean the core of one of the cycles $\left\{c_{e_{1}, T}, \ldots, c_{e_{n}, T}\right\}$. Note that since the graph $\Gamma$ has no vertices of valence one and since $T$ is a maximal subtree of $\Gamma$, every edge of $\Gamma$ belongs to the (core of at least one) cycle $c_{e_{i}, T}$.

Note that two different conjugates of $\Gamma$, say $\Gamma^{g}$ and $\Gamma^{h}$, share at most the star of a vertex $u_{g, h}$. That is, $u_{g, h} \in V\left(\Gamma^{g}\right) \cap V\left(\Gamma^{h}\right)$ and $\Gamma^{g} \cap \Gamma^{h} \subset \operatorname{Star}\left(u_{g, h}\right)$; see Lemma 19.

Claim 1 If the vertex $u_{g, h}$ belongs to $\varphi(\Gamma)$, then either $\varphi(\Gamma) \cap\left(\Gamma^{g} \backslash \Gamma^{h}\right)=\varnothing$ or $\varphi(\Gamma) \cap\left(\Gamma^{h} \backslash \Gamma^{h}\right)=\varnothing$.

Indeed, if $\varphi(\Gamma)$ intersects $\Gamma^{g}$ and $\Gamma^{h}$ outside the star $\operatorname{Star}\left(u_{g, h}\right)$ then, by Lemma 19, $\operatorname{Star}\left(u_{g, h}\right)$ separates the extension graph $\Gamma^{e}$ (and $\Gamma^{g}$ and $\Gamma^{h}$ belong to different connected components), so $\operatorname{Star}\left(u_{g, h}\right)$ also separates the image $\varphi(\Gamma) \simeq \Gamma$. However, we assume $\Gamma$ to be atomic, and so, in particular, it does not contain vertices with closed separating stars. 
Claim 2 Let $c$ be a cycle in $\Gamma$, and assume that $\varphi(c)<\Gamma^{e}$ is not contained in one conjugate of $\Gamma$, that is, $\varphi(c) \cap \Gamma^{g} \neq \varphi(c)$ for all $g \in \mathbb{G}(\Gamma)$. Then $\varphi(c)$ is contained in the union of cycles $c_{i}^{g_{i}}<\Gamma^{g_{i}}$ for $g_{i} \in \mathbb{G}(\Gamma)$, and the length of each $c_{i}$ is strictly less than the length of $\varphi(c)$.

Indeed, since $\varphi(c)$ is not contained in one conjugate of $\Gamma$, it follows that there exist $v, w \in \varphi(c)$ and $g, h \in \mathbb{G}(\Gamma)$ such that $v \in \Gamma^{g} \backslash \Gamma^{h}, w \in \Gamma^{h} \backslash \Gamma^{g}$ and $\Gamma^{g} \cap \Gamma^{h} \neq \varnothing$. Since $\Gamma^{g} \cap \Gamma^{h} \neq \varnothing$, it follows from Lemma 19 that there exists $u_{g, h}$ such that its star $\operatorname{Star}\left(u_{g, h}\right)$ separates $\Gamma^{g}$ and $\Gamma^{h}$ and so separates $\varphi(c)$. Furthermore, it follows from Claim 1 that $u_{g, h} \notin \varphi(c)$. Let $d_{1}, \ldots, d_{k}$, for $k \geq 2$, be the connected components of $\varphi(c) \backslash \operatorname{Star}\left(u_{g, h}\right)$. For each $d_{i}$, there is a path $p_{i}$ in the star of $u_{g, h}$ of length at most 2 such that $d_{i} \cup p_{i}$ is a cycle $c_{i}$ in $\Gamma^{e}$. Since $\Gamma$ is atomic, it follows that there are no squares or triangles in $\Gamma^{e}$, and so we have that $\left|c_{i}\right|=\left|d_{i} \cup p_{i}\right| \geq 5$. Furthermore, since $\left|p_{i}\right| \leq 2$, it follows that $\left|d_{i}\right| \geq 3$. We conclude that, since $k \geq 2$ and $\left|d_{i}\right| \geq 3$, $\left|c_{i}\right|=\left|d_{i}\right|+\left|p_{i}\right| \leq\left|d_{i}\right|+2<\left|d_{1}\right|+\cdots+\left|d_{k}\right|=|\varphi(c)|=|c|$.

Claim 3 If $\Gamma^{\prime}$ is a full subgraph of $\Gamma, c$ is a cycle of minimal length in $\Gamma, \Gamma^{\prime}$ and $c$ intersect at least in an edge $e$ and $\varphi\left(\Gamma^{\prime}\right)$ is contained in a conjugate of $\Gamma$, say $\varphi\left(\Gamma^{\prime}\right)<\Gamma^{g}$, then $\varphi(c)$ is also contained in $\Gamma^{g}$, ie $\varphi\left(\Gamma^{\prime} \cup c\right)<\Gamma^{g}$.

Indeed, since $c$ is of minimal length, it follows from Claim 2 that $\varphi(c)$ is contained in one conjugate of $\Gamma$. Assume towards contradiction that $\varphi(c)<\Gamma^{h}$ and $\Gamma^{h} \neq \Gamma^{g}$. Since $\Gamma^{\prime}$ and $c$ share at least the edge $e$, it follows that $\varphi(e) \in \Gamma^{g} \cap \Gamma^{h}$. Since conjugates of $\Gamma$ share at most a star of a vertex, it follows that $u_{g, h}$ is a vertex of $e$ and so belongs to $\varphi(\Gamma)$, contradicting Claim 1. Hence, $\varphi\left(\Gamma^{\prime} \cup c\right)<\Gamma^{g}$ for some $g \in \mathbb{G}(\Gamma)$.

Let $c$ be a cycle in $\Gamma$. Define the neighbourhood $N(c)$ of the cycle $c$ to be the collection of all cycles in $\Gamma$ that share at least one edge with $c$. Recall that by cycles in $\Gamma$ we mean marked cycles. We say that a connected subgraph $S$ of $\Gamma$ is a component if it is a union of cycles and has no cut-points. The neighbourhood $N(S)$ of a component $S$ is the union of neighbourhoods of all the cycles which belong to $S$. Note that, by definition, the neighbourhood of a component is itself a component.

Let $S$ be a component. Given a cycle $c$ in $S$, the complexity $\operatorname{comp}(c)$ of $c$ in $S$ is

$$
\operatorname{comp}(c)=\left(r_{5}, \ldots, r_{M}\right) \in \mathbb{N}^{M-4},
$$

where $r_{l}$ is the number of cycles of length $l$ that belong to $N(c) \cap S$ for $l=5, \ldots, M$ and $M=\left|c_{T, e_{n}}\right|$. Define the finite set of complexities of cycles in $S$ as

$$
\mathcal{K}(S)=\{\operatorname{comp}(c) \mid \mathrm{c} \text { is a cycle in } \mathrm{S}\}
$$


and $\mathcal{K}$ to be the union of complexities $\mathcal{K}(S)$ over all components $S$.

To a component $S$, we associate the tuple

$$
\left(m_{l, \mathrm{comp}_{i}}\right)_{5 \leq l \leq M, \mathrm{comp}_{i} \in \mathcal{K}}^{S}
$$

where $m_{l, \text { comp }_{i}}$ is the number of cycles in $S$ of length $l$ and complexity comp (in $S$ ) ordered lexicographically from minimal to maximal length and from maximal to minimal complexity. That is, the position $\left(k, \mathrm{comp}_{i}\right)$ in the tuple is before the position $\left(k^{\prime}, \operatorname{comp}_{i}^{\prime}\right)$ if either $k<k^{\prime}$ or both $k=k^{\prime}$ and $\operatorname{comp}_{i}>\operatorname{comp}_{i}^{\prime}$. If no confusion arises, we drop the subindices and denote the tuple simply by $\left(m_{l, \text { comp }_{i}}\right)^{S}$.

The lexicographical order on the tuples $\left(m_{l, \text { comp }_{i}}\right)^{S}$ naturally defines an ordering $\prec$ on the components of $\Gamma: S^{\prime} \prec S$ if an only if $\left(m_{l, \text { comp }_{i}}\right)^{S}<\left(m_{l, \text { comp }_{i}}\right)^{S^{\prime}}$ in the lexicographical order, that is, there exists $\left(l, \operatorname{comp}_{j}\right)$ such that $m_{k, \text { comp }_{i}}(S)=$ $m_{k, \text { comp }_{i}}\left(S^{\prime}\right)$ for all $\left(k, \operatorname{comp}_{i}\right)<\left(l, \operatorname{comp}_{j}\right)$ and $m_{l, \mathrm{comp}_{j}}\left(S^{\prime}\right)<m_{l, \mathrm{comp}_{j}}(S)$. In this ordering, a component $S$ is maximal if it contains the maximal number of cycles of minimal length and maximal complexity.

Let $c$ be a cycle of minimal length in $\Gamma$. Define the minimal component of $c$ to be the maximal connected subgraph $C$ of $\Gamma$ containing $c$ so that $C$ is a union of cycles of minimal length in $\Gamma$ without cut points. Let $\mathcal{S}_{1}$ be the set of minimal components of cycles of minimal length in $\Gamma$ which are maximal with respect to the order $\prec$.

We define the components $\mathcal{S}_{q}$ recursively as follows. Consider the set $\mathcal{T}_{q}=\left\{N\left(S_{q-1}\right) \mid\right.$ $\left.S_{q-1} \in \mathcal{S}_{q-1}\right\}$ of neighbourhoods of components of $\mathcal{S}_{q-1}$. Define $\mathcal{S}_{q}$ to be the set of maximal components of $\mathcal{T}_{q}$ with respect to the order $\prec$. Note that, for $q$ large enough, we have that $S_{q}=\Gamma$ for all $S_{q} \in \mathcal{S}_{q}$. We prove by induction on $q$ that, for all $S_{q} \in \mathcal{S}_{q}$, $\varphi\left(S_{q}\right)<\Gamma^{g}$ for some $g \in \mathbb{G}(\Gamma)$.

Induction base Let $S_{1} \in \mathcal{S}_{1}$. By definition of $S_{1}$ and by recursively applying Claim 3, it follows that $\varphi\left(S_{1}\right)$ is contained in $\Gamma^{g}$ for some $g \in \mathbb{G}(\Gamma)$. Since $\varphi$ is a monomorphism of graphs, $\varphi\left(S_{1}\right)$ is a component of $\Gamma^{g}$. Since $S_{1}$ is maximal in the sense of $\prec$, since $\varphi\left(S_{1}\right) \subseteq \Gamma^{g} \simeq \Gamma$ and since $\varphi$ is an embedding, it follows that the complexity of the image $\operatorname{comp}(\varphi(c))$ in $\varphi\left(S_{1}\right)$ is equal to the complexity $\operatorname{comp}(c)$ in $S_{1}$ and $\varphi\left(S_{1}\right) \in \mathcal{S}_{1}\left(\Gamma^{g}\right)$. In other words, $\varphi$ induces a permutation on the set of components in $\mathcal{S}_{1}$ that preserves the complexity of the cycles, ie $\prec-$ maximal minimal components in $\Gamma$ are mapped by $\varphi$ to $\prec-$ maximal minimal components in $\Gamma^{e}$, and $\varphi$ preserves complexity of cycles from components in $\mathcal{S}_{1}$. To simplify the notation, without loss of generality, we shall assume that $g=1$.

Induction step To prove the induction step, we proceed by induction on the complexity of the cycles $d$ that belong to $S_{q}$ but not to $S_{q-1}$, denoted $d \in S_{q} \backslash S_{q-1}$, to show that the image of $S_{q}$ under $\varphi$ belongs to $\Gamma$. 
Let $d$ be a cycle in $S_{q} \backslash S_{q-1}$ of minimal length and maximal complexity. By the induction hypothesis, we have that $\varphi\left(S_{q-1}\right)=S_{q-1}^{\prime} \in \mathcal{S}_{q-1}$ where $S_{q-1}^{\prime} \subseteq \Gamma<\Gamma^{e}$. Since $\varphi$ is a graph monomorphism, $\varphi(d) \cap S_{q-1}^{\prime}=\varphi\left(d \cap S_{q-1}\right)$, and so $\varphi(d)$ belongs to the neighbourhood of $S_{q-1}^{\prime}$. Since $d$ has minimal length in $S_{q}$, it follows from Claim 3 that $\varphi(d)=d^{\prime}$ belongs to $\Gamma<\Gamma^{e}$, and so $d^{\prime}$ belongs $N\left(S_{q-1}^{\prime}\right) \cap \Gamma=S_{q}^{\prime}$.

Furthermore, since $S_{q}$ is $\prec-$ maximal in $\mathcal{T}_{q}, \varphi(d) \subseteq \Gamma, \varphi$ is an embedding and $d$ has maximal complexity in $S_{q} \backslash S_{q-1}$, it follows that $d^{\prime}$ has maximal complexity in $N\left(S_{q-1}^{\prime}\right) \cap \Gamma$. We conclude that the bijection $\varphi$ on $S_{q-1}$ extends to a bijection between cycles of minimal length in $S_{q} \backslash S_{q-1}$ and $S_{q}^{\prime} \backslash S_{q-1}^{\prime}$, and that cycles of maximal complexity in $S_{q} \backslash S_{q-1}$ are mapped to cycles of maximal complexity in $S_{q}^{\prime} \backslash S_{q-1}^{\prime}$. Hence, all cycles of minimal length in the neighbourhood of $\varphi\left(S_{q-1}\right)$ belong to the image of $\varphi\left(S_{q}\right)$. This proves the base of induction.

Assume by induction that $\varphi$ is a bijection between $S_{q-1}$ and $S_{q-1}^{\prime}$, as well as between the cycles in $S_{q} \backslash S_{q-1}$ and $S_{q}^{\prime} \backslash S_{q-1}^{\prime}$ of length less than or equal to $l-1$, and between cycles of length $l$ and complexity greater than $K$. Let $c$ be a cycle in $S_{q} \backslash S_{q-1}$ of length $l$ and maximal complexity less than $K$. It follows from Claim 2 that either $\varphi(c)=c^{\prime} \in S_{q}^{\prime} \backslash S_{q-1}^{\prime}$ and $\operatorname{comp}(c)=\operatorname{comp}\left(c^{\prime}\right)$, or $\varphi(c)$ is contained in the image of shorter cycles and one of them belongs to the neighbourhood of $S_{q-1}^{\prime}$. However, by induction, all the cycles in $S_{q}^{\prime} \backslash S_{q-1}^{\prime}$ of length less than $l$, or of length $l$ and complexity greater than $K$, are in one-to-one correspondence with the cycles of the same complexity in $S_{q} \backslash S_{q-1}$. In particular, these cycles belong to the image of $\varphi\left(S_{q}\right)$. This implies that the $\varphi(c)$ is not contained in the image of shorter cycles, and so we deduce that $\varphi(c)=c^{\prime} \in S_{q}^{\prime} \backslash S_{q-1}^{\prime}$. Moreover, since $S_{q}$ is $\prec-$ maximal in $\mathcal{T}_{q}$, $\varphi(c) \in S_{q}^{\prime} \backslash S_{q-1}^{\prime}, \varphi$ is an embedding, and $c$ has maximal complexity (in $S_{q}$ ) less than $K$ among all cycles in $S_{q} \backslash S_{q-1}$, we conclude that $\operatorname{comp}\left(c^{\prime}\right)=\operatorname{comp}(c)$. So, we get a bijection between the cycles in $S_{q} \backslash S_{q-1}$ and $S_{q}^{\prime} \backslash S_{q-1}^{\prime}$ of length $l$ and maximal complexity less than $K$. This finishes the proof of the induction step.

The statement follows, since $S_{q}=\Gamma$ for all large enough $q$.

Theorem 21 Let $\Delta$ and $\Gamma$ be two atomic graphs. Then $\mathbb{G}(\Delta)$ and $\mathbb{G}(\Gamma)$ embed into each other if and only if $\Delta=\Gamma$.

Proof Since atomic graphs are triangle-free, it follows from [17] that $\mathbb{G}(\Delta)<\mathbb{G}(\Gamma)$ (resp. $\mathbb{G}(\Gamma)<\mathbb{G}(\Delta)$ ) if and only if there is a graph embedding $\varphi: \Delta<\Gamma^{e}$ (resp. $\left.\psi: \Delta<\Gamma^{e}\right)$.

Let $V(\Delta)=\left\{a_{1}, \ldots, a_{l}\right\}$ and $V(\Gamma)=\left\{b_{1}, \ldots, b_{n}\right\}$. Let

$$
\varphi: a_{i} \mapsto b_{f(i)}^{w_{i}\left(b_{1}, \ldots, b_{n}\right)}
$$


and

$$
\psi: b_{j} \mapsto a_{g(j)}^{v_{j}\left(a_{1}, \ldots, a_{l}\right)},
$$

where $w_{i} \in \mathbb{G}(\Gamma)$ and $v_{j} \in \mathbb{G}(\Delta)$ for $i=1, \ldots, l$ and $j=1, \ldots, n$. We observe that $\varphi$ and $\psi$ induce homomorphisms $\bar{\varphi}: \mathbb{G}(\Delta) \rightarrow \mathbb{G}(\Gamma)$ and $\bar{\psi}: \mathbb{G}(\Gamma) \rightarrow \mathbb{G}(\Delta)$. By [17], there exists $N \in \mathbb{N}$ depending only on $\Gamma$ and $\Delta$ so that the homomorphisms $\varphi^{*}$ and $\psi^{*}$ induced by the maps

$$
a_{i} \mapsto\left(b_{f(i)}^{N}\right)^{w_{i}} \quad \text { and } \quad b_{j} \mapsto\left(a_{g(j)}^{N}\right)^{v_{j}},
$$

correspondingly, are group monomorphisms.

Furthermore, the maps $\bar{\varphi}$ and $\bar{\psi}$ naturally induce embeddings $\varphi^{\prime}: \Delta^{e} \rightarrow \Gamma^{e}$ and $\psi^{\prime}: \Gamma^{e} \rightarrow \Delta^{e}$ as follows. In order to define $\varphi^{\prime}$ and $\psi^{\prime}$, it suffices to determine the images of the vertices of the extension graphs. By definition of the extension graph, their vertices are labelled by $a_{i}^{u_{i}}$ and $b_{j}^{q_{j}}$, correspondingly. Set

$$
\varphi^{\prime}: a_{i}^{u_{i}} \mapsto b_{f(i)}^{\varphi^{*}\left(u_{i}\right)} \quad \text { and } \quad \psi^{\prime}: b_{j}^{q_{j}} \mapsto a_{g(j)}^{\psi^{*}\left(q_{j}\right)} .
$$

Note that $\varphi^{\prime}$ and $\psi^{\prime}$ are graph embeddings, since so are $\varphi$ and $\psi$ and since $\varphi^{*}$ and $\psi^{*}$ are group embeddings.

By Theorem 20, the embedding $\varphi \psi^{\prime}$ of $\Delta$ into $\Delta^{e}$ is unique up to conjugacy and graph automorphism. It follows that if $i_{1} \neq i_{2}$, then $a_{g\left(f\left(i_{1}\right)\right)} \neq a_{g\left(f\left(i_{2}\right)\right)}$. Hence, we have that $f\left(i_{1}\right) \neq f\left(i_{2}\right)$, and we conclude that $|V(\Delta)| \leq|V(\Gamma)|$. Moreover, since $\varphi$ is a graph embedding, it follows that if $\left(a_{i_{1}}, a_{i_{2}}\right)$ is an edge of $\Delta$, then $\left(b_{f\left(i_{1}\right)}, b_{f\left(i_{2}\right)}\right)$ is an edge of $\Gamma$. We conclude that $\Delta$ is a subgraph of $\Gamma$.

An analogous argument for $\psi \varphi^{\prime}$ shows that $\Gamma$ is a subgraph of $\Delta$. Therefore, $\Delta$ and $\Gamma$ are isomorphic graphs.

Often, the quasi-isometric rigidity of the group is closely related with the group being co-Hopfian, a property which can be viewed as some type of algebraic rigidity. For instance, one of the consequences of Mostow rigidity is that irreducible lattices in semisimple Lie groups are co-Hopfian (with the exception of free groups). Recall that a group is termed co-Hopfian if it satisfies the following equivalent conditions:

- It is not isomorphic to any proper subgroup.

- Every injective endomorphism of the group is an automorphism.

Besides this classical example, there are many other interesting co-Hopfian groups: nonelementary freely indecomposable torsion-free word-hyperbolic groups; the fundamental groups of finite volume pinched negatively curved manifolds of dimension 
greater than 2; closed aspherical manifolds $M$ with $i(M) \neq 0$, where $i$ is a homotopy invariant of closed manifolds which is multiplicative under finite covers (eg Euler characteristic, signature, simplicial volume, or $L^{2}$-Betti number); finitely generated torsion-free nilpotent groups with a specific automorphism group of their Lie algebra, etc; see [6] and references there. Our next goal is to show that the quasi-isometric rigidity of the class of atomic pc groups is also related to the co-Hopfian property of the $\mathbb{Q}$-completion of these groups.

Let us first show that injective endomorphisms of an atomic pc group are rigid; that is, any embedding $\varphi: \mathbb{G}(\Gamma) \rightarrow \mathbb{G}(\Gamma)$ is the identity up to an automorphism and taking powers of the generators.

Corollary 22 Let $\Gamma$ be an atomic graph and $\psi: \mathbb{G}(\Gamma) \rightarrow \mathbb{G}(\Gamma)$ an injective endomorphism. Then there exist $g \in \mathbb{G}(\Gamma), \sigma \in \operatorname{Aut}(\Gamma)$ and $k_{v} \in \mathbb{Z} \backslash 0$ such that, for all $v \in V(\Gamma)$, we have that

$$
\psi(v)=g^{-1} \sigma(v)^{k_{v}} g, \quad v \in V(\Gamma) .
$$

In other words, $\psi$ is, up to conjugacy, graph automorphism and taking powers, the identity endomorphism.

Proof Let $v \in V(\Gamma)$. Since $\Gamma$ is an atomic graph, it follows that the centraliser $C(v)$ of $v$ is isomorphic to $\mathbb{Z} \times F$ where $F$ is a free group of rank greater than or equal to 2. On the other hand, it follows from the description of centralisers in pc groups that if $C(h)$ is isomorphic to $\mathbb{Z} \times F$, where $h \in \mathbb{G}(\Gamma)$ and $F$ is a free group of rank greater than or equal to 2 , then $h$ is conjugate of a power of a generator, that is, $h=\left(v^{k}\right)^{g}$ for $k \in \mathbb{Z}$ and $g \in \mathbb{G}(\Gamma)$. Therefore, for all $v \in V(\Gamma)$, we have that $\psi(v)=\left(w_{v}^{k_{v}}\right)^{g_{v}}$ where $w_{v} \in V(\Gamma), k_{v} \in \mathbb{Z}$ and $g_{v} \in \mathbb{G}(\Gamma)$. Since $\psi$ is an embedding, we have that $\left\{w_{v}^{g_{v}} \mid v \in V(\Gamma)\right\}$, viewed as vertices of $\Gamma^{e}$, spans an induced subgraph isomorphic to $\Gamma$. Thus, the map $v \rightarrow w_{v}^{g_{v}}$ induces a graph embedding $\varphi$ from $\Gamma$ to the extension graph $\Gamma^{e}$. By Theorem 20, up to conjugacy and graph automorphism, there is only one graph embedding induced by the identity map. So, $w_{v}^{g}=w_{v}^{g}$ for all $v \in V(\Gamma)$, and $v \rightarrow w_{v}$ induces a graph automorphism $\sigma \in \operatorname{Aut}(\Gamma)$. We conclude that

$$
\psi(v)=g^{-1} \sigma(v)^{k_{v}} g, \quad v \in V(\Gamma),
$$

for some $g \in \mathbb{G}(\Gamma), \sigma \in \operatorname{Aut}(\Gamma)$ and $k_{v} \in \mathbb{Z} \backslash 0$.

In the context of the curve complex of a surface, this type of rigidity for embeddings from pc groups to modular groups was proven by Aramayona and Souto in [3]; see also [2]. More precisely, the authors show that, for some rigid finite sets $X$ of the curve complex $\mathcal{C}(S)$ of a surface $S \neq S_{1,2}$, every injective homomorphism from the 
pc group $\mathbb{G}(X)$ into the modular group $\operatorname{Mod}^{ \pm}(S)$ is obtained, up to conjugation, by taking powers of roots of Dehn twists in the vertices of $X$.

Corollary 22 shows that the only reason why atomic pc groups are not co-Hopfian is that the injective endomorphisms induced by the maps $v \rightarrow v^{k_{v}}$ that send generators to proper powers of themselves are not automorphisms. We now pass to a divisible extension of the atomic pc group, its $\mathbb{Q}$-completion, to assure that these injective endomorphisms induce automorphisms there, and we show that the $\mathbb{Q}$-completions of atomic pc groups are co-Hopfian.

One of the classical theorems in the theory of abelian groups asserts that every abelian group can be embedded into a divisible abelian group. An analogous result for torsionfree locally nilpotent groups was proven by Mal'cev. Since then, many mathematicians, such as Kontorivich, Hall and Baumslag, have studied the classes of groups for which there always exists the $n^{\text {th }}$ root of an element as well as groups for which such an $n^{\text {th }}$ root is unique. Further developing ideas of Lyndon in this context, Miasnikov and Remeslennikov showed that free groups also embed into a divisible group, the free $\mathbb{Q}$-group, and they described its algebraic structure; see [21].

In the same spirit, we prove in [9] that every pc group $\mathbb{G}(\Gamma)$ embeds into a divisible group and that the category of divisible $\mathbb{G}(\Gamma)$-groups has an initial object, the $\mathbb{Q}$ completion $\mathbb{G}(\Gamma)^{\mathbb{Q}}$ of $\mathbb{G}(\Gamma)$. Furthermore, as in the case of free groups, the $\mathbb{Q}$ completion $\mathbb{G}(\Gamma)^{\mathbb{Q}}$ can be described algebraically as an iterated sequence of extensions of centralisers of elements.

Since the construction of the $\mathbb{Q}$-completion is technically involved, for readers not familiar with this notion, we consider an intermediate group, denoted by $\mathcal{G}(\Gamma, \mathbb{Q})$, which is a subgroup of the $\mathbb{Q}$-completion $\mathbb{G}(\Gamma)^{\mathbb{Q}}$ and the smallest extension of $\mathbb{G}(\Gamma)$ which is co-Hopfian (when the graph $\Gamma$ is atomic). Given a simplicial graph $\Gamma$, we define the group $\mathcal{G}(\Gamma, \mathbb{Q})$ as the graph product with underlying graph $\Gamma$ and vertex groups isomorphic to $\mathbb{Q}$. Note that we view $\mathbb{Q}$ as a divisible abelian group; that is, in exponential notation, we have that for all $n \in \mathbb{N}$ and for all $x \in \mathbb{Q}$, there exists $y \in \mathbb{Q}$ such that $y^{n}=x$. Moreover, we identify elements $q$ from the vertex group $\mathbb{Q}$ associated to $v$ with $v^{q}$. This way, the ring $\mathbb{Q}$ has a natural action on the vertex groups: given $p \in \mathbb{Q}$ (viewed as a ring) and $v^{q} \in \mathbb{Q}$ (an element of the vertex group), we define the action of $p$ on $v^{q}$ as $v^{p q}$. The defined group is not divisible, as only products of pairwise commuting elements of the vertex groups have $n^{\text {th }}$ roots for all $n \in \mathbb{N}$. It is not difficult to see that $\mathbb{G}(\Gamma)<\mathcal{G}(\Gamma, \mathbb{Q})<\mathbb{G}(\Gamma)^{\mathbb{Q}}$.

Corollary 23 Let $\Gamma$ be an atomic graph. Then $\mathcal{G}(\Gamma, \mathbb{Q})$ (resp. the $\mathbb{Q}$-completion $\mathbb{G}(\Gamma)^{\mathbb{Q}}$ of $\left.\mathbb{G}(\Gamma)\right)$ is co-Hopfian. 
Proof It suffices to notice that if the centraliser $C(h)$ is nonabelian if and only if it is isomorphic to $\mathbb{Q} \times F$, where $F$ is the free product $\mathbb{Q}^{(1)} * \cdots * \mathbb{Q}^{(n)}$ for $n \geq 2$, if and only if $h$ is a conjugate of an element of a vertex group, that is, $h=g^{-1} v^{q} g$, where $v^{q}$ belongs to the vertex group $v, q \in \mathbb{Q}$ and $g \in \mathcal{G}(\Gamma, \mathbb{Q})$.

For the $\mathbb{Q}$-completion $\mathbb{G}(\Gamma)^{\mathbb{Q}}$, we have that the centraliser $C(h)$ is nonabelian if and only if $C(h) \simeq \mathbb{Q} \times F^{\mathbb{Q}}$, where $F^{\mathbb{Q}}$ is the $\mathbb{Q}$-completion of a (nonabelian) free group, if and only if $h$ is a conjugate of a $\mathbb{Q}$-power of a generator, that is, $h=g^{-1}\left(v^{q}\right) g$, where $v \in V(\Gamma), q \in \mathbb{Q}$, and $g \in \mathbb{G}(\Gamma)^{\mathbb{Q}}$.

The rest of the proof is analogous to the proof of Corollary 22.

Note that, for arbitrary graphs, the groups $\mathcal{G}(\Gamma, \mathbb{Q})$ and $\mathbb{G}(\Gamma)^{\mathbb{Q}}$ are far from being co-Hopfian. It suffices to take $\Gamma$ to be the edgeless graph with more than one vertex; for a connected example, take $\Gamma$ to be a path of length greater than 2 .

We can reformulate these observations in yet another way as follows.

Corollary 24 Let $\Gamma$ be an atomic graph. Then each injective endomorphism

$$
\psi: \mathbb{G}(\Gamma) \rightarrow \mathbb{G}(\Gamma)
$$

extends to automorphisms $\psi^{\prime}$ of $\mathcal{G}(\Gamma, \mathbb{Q})$ and $\psi^{\prime \prime}$ of $\mathbb{G}(\Gamma)^{\mathbb{Q}}$, ie one has the following commutative diagram:

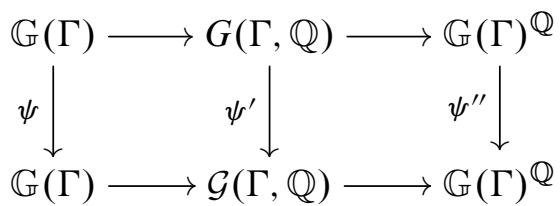

\section{Concluding remarks}

The main motivation that brought us to formulate Conjecture 2 comes, in fact, from a stronger statement about asymptotic cones of pc groups. The study of asymptotic geometry of pc groups and, in particular, the structure of their asymptotic cones, led us to believe that asymptotic cones of pc groups can be classified up to bilipschitz equivalence in terms of their extension graphs. More precisely, we raise the following question.

Question 25 Given simplicial graphs $\Gamma$ and $\Delta$, is it true that the asymptotic cones of $\mathbb{G}(\Gamma)$ and $\mathbb{G}(\Delta)$ are bilipschitz equivalent if and only if $\Gamma<\Delta^{e}$ and $\Delta<\Gamma^{e}$ ? 
Since asymptotic cones of quasi-isometric pc groups are bilipschitz equivalent, beside being extremely interesting on its own right, Conjecture 2 can be viewed as a weaker formulation, and an ideal test case, of Question 25.

As we mentioned in the introduction, Question 1 has a positive answer when we restrict to commensurable pc groups. Recall that two groups $G$ and $G^{\prime}$ are (abstractly) commensurable if they have isomorphic finite index subgroups, that is, if there exist $H<_{f i} G$ and $H^{\prime}<_{f i} G^{\prime}$ such that $H \simeq H^{\prime}$. In particular, groups that are commensurable are quasi-isometric. Although the following result is easy to prove, it is worth mentioning and we record it as a lemma.

Lemma 26 If the pc groups $\mathbb{G}(\Delta)$ and $\mathbb{G}(\Gamma)$ are commensurable, then $\mathbb{G}(\Gamma)<\mathbb{G}(\Delta)$ and $\mathbb{G}(\Delta)<\mathbb{G}(\Gamma)$.

Proof Assume that $\mathbb{G}(\Delta)$ and $\mathbb{G}(\Gamma)$ are commensurable. By definition, there exist finite index subgroups $H<_{f i} \mathbb{G}(\Gamma)$ and $K<_{f i} \mathbb{G}(\Delta)$ such that $H \simeq K$. Since the subgroups $H$ and $K$ are of finite index, there exists $N \in \mathbb{N}$ so that the subgroup generated by the $N^{\text {th }}$ powers of the generators of $\mathbb{G}(\Delta)$ (resp. of $\mathbb{G}(\Gamma)$ ) is a subgroup of $H$ (resp. of $K$ ):

$$
\begin{gathered}
\left\langle x_{1}^{N}, \ldots, x_{n}^{N} \mid x_{i} \in V(\Gamma)\right\rangle<H, \\
\left\langle y_{1}^{N}, \ldots, y_{m}^{N} \mid y_{i} \in V(\Delta)\right\rangle<K .
\end{gathered}
$$

By [19], the subgroup $\left\langle x_{1}^{N}, \ldots, x_{n}^{N} \mid x_{i} \in V(\Gamma)\right\rangle$ is isomorphic to $\mathbb{G}(\Gamma)$, and also $\left\langle y_{1}^{N}, \ldots, y_{m}^{N} \mid y_{i} \in V(\Delta)\right\rangle$ is isomorphic to $\mathbb{G}(\Delta)$. Using these isomorphisms we have that $\mathbb{G}(\Delta) \hookrightarrow H \simeq K<\mathbb{G}(\Gamma)$ and vice-versa. Therefore, if $\mathbb{G}(\Gamma)$ is commensurable to $\mathbb{G}(\Delta)$, then $\mathbb{G}(\Gamma)<\mathbb{G}(\Delta)$ and $\mathbb{G}(\Delta)<\mathbb{G}(\Gamma)$.

Since Question 1 has a positive answer for commensurable pc groups, one may wonder why we state Conjecture 2 using embeddability in the extension graph, rather than just embeddability of groups as in Question 1. The motivation comes from the study of pc groups $\mathbb{G}(\Gamma)$ and $\mathbb{G}(\Delta)$ for which there is an embedding of $\mathbb{G}(\Delta)$ to $\mathbb{G}(\Gamma)$ but no graph embeddings of $\Delta$ to the extension graph $\Gamma^{e}$; see [10]. The nature of such embeddings indicates that the corresponding groups $\mathbb{G}(\Delta)$ and $\mathbb{G}(\Gamma)$ are not commensurable (in fact, they seem to be not quasi-isometric). This brought us to believe that if Question 1 has a positive answer, then so does Conjecture 2. Therefore, a good starting point to check if Conjecture 2 is indeed a consequence of Question 1 would be to answer the following question, which is also of an independent interest.

Question 27 If $\mathbb{G}(\Delta)$ and $\mathbb{G}(\Gamma)$ are commensurable (and so $\mathbb{G}(\Delta)<\mathbb{G}(\Gamma)$ and $\mathbb{G}(\Gamma)<\mathbb{G}(\Delta))$, does it follow that $\Delta<\Gamma^{e}$ and $\Gamma<\Delta^{e}$ ? 
In some cases (for instance, if the graph $\Gamma$ (or $\Delta$ ) is triangle-free, triangle-built, a tree or the complement of a tree), the embeddability between pc groups is equivalent to the graph embeddability in to the extension graph. That is, $\mathbb{G}(\Delta)<\mathbb{G}(\Gamma)$ and $\mathbb{G}(\Gamma)<\mathbb{G}(\Delta)$ if and only if $\Delta<\Gamma^{e}$ and $\Gamma<\Delta^{e}$; see [17], [10] and [8]. Therefore, in these cases, Question 27 has a positive answer. Moreover, if one further assumes $\Gamma$ to be a triangle and square-free graph without any degree-one or degree-zero vertex, then if $\mathbb{G}(\Delta)$ and $\mathbb{G}(\Gamma)$ are commensurable, we have that the corresponding extension graphs are isomorphic, ie $\Delta^{e} \simeq \Gamma^{e}$; see [18, Proposition 7].

In the cases of quasi-isometric classification of pc groups that we analyse, we show not only that Conjecture 2 holds, but also so does its converse. However, we would like to stress that graph embeddability into the extension graph is not a sufficient condition to assure quasi-isometry of the corresponding groups. One obvious obstruction is that the graphs $\Gamma$ and $\Delta$ both need to be either connected or disconnected since the number of ends is a quasi-isometry invariant; see [22]. Similarly, since the cyclic JSJ is also a quasi-isometry invariant, one can find connected examples for which the converse of Conjecture 2 does not hold: consider the connected union of two cycles $C_{5}$ of length 5 where the cycles only share a vertex $p$, and denote this graph by $C_{5} \vee_{p} C_{5}$. It is easy to show that $C_{5} \vee_{p} C_{5}$ embeds into the extension graph of $C_{5}$ and vice-versa. However, the corresponding pc groups are not quasi-isometric since $C_{5} \vee_{p} C_{5}$ has a nontrivial JSJ-decomposition over the infinite cyclic group generated by $p$, while $C_{5}$ does not; see [11].

Finally, we also mentioned that a positive answer to Conjecture 2 would imply that coherence is a quasi-isometric invariant in the class of pc groups: if the pc group $\mathbb{G}(\Gamma)$ is coherent and the pc group $\mathbb{G}(\Delta)$ is quasi-isometric to $\mathbb{G}(\Gamma)$, then $\mathbb{G}(\Delta)$ is coherent. This is a consequence of the following remark.

Remark 28 In [12], Droms gives a graph theoretical characterisation of the class of coherent pc groups: a pc group $\mathbb{G}(\Gamma)$ is coherent if and only if $\Gamma$ is chordal, ie $\Gamma$ does not contain cycles of length greater than or equal to 4 . If $\Gamma$ is chordal, then, as shown in [17], the extension graph $\Gamma^{e}$ and any induced subgraph of $\Gamma^{e}$ are also chordal.

Acknowledgements The author is supported by the Juan de la Cierva Programme and IKEBASQUE Basque Foundation for Science; and partly supported by the ERC grant PCG-336983, the Spanish Government grant MTM2014-53810-C2-2-P and the Basque Government grant IT974-16. 


\section{References}

[1] A R Ahlin, The large scale geometry of products of trees, Geom. Dedicata 92 (2002) 179-184 MR1934017

[2] J Aramayona, C J Leininger, Finite rigid sets in curve complexes, J. Topol. Anal. 5 (2013) 183-203

[3] J Aramayona, J Souto, A remark on homomorphisms from right-angled Artin groups to mapping class groups, C. R. Math. Acad. Sci. Paris 351 (2013) 713-717

[4] J A Behrstock, T Januszkiewicz, W D Neumann, Quasi-isometric classification of some high dimensional right-angled Artin groups, Groups Geom. Dyn. 4 (2010) 681692 MR2727658

[5] J A Behrstock, W D Neumann, Quasi-isometric classification of graph manifold groups, Duke Math. J. 141 (2008) 217-240 MR2376814

[6] I Belegradek, On co-Hopfian nilpotent groups, Bull. London Math. Soc. 35 (2003) 805-811 MR2000027

[7] M Bestvina, B Kleiner, M Sageev, The asymptotic geometry of right-angled Artin groups, I, Geom. Topol. 12 (2008) 1653-1699 MR2421136

[8] M Casals-Ruiz, Embeddability and universal theory of partially commutative groups, Int. Math. Res. Not. 2015 (2015) 13575-13622

[9] M Casals-Ruiz, A Duncan, I Kazachkov, Lyndon's completion for partially commutative groups, preprint

[10] M Casals-Ruiz, A Duncan, I Kazachkov, Embedddings between partially commutative groups: two counterexamples, J. Algebra 390 (2013) 87-99

[11] M Clay, When does a right-angled Artin group split over $\mathbb{Z}$ ?, preprint (2014) arXiv: 1403.1842

[12] C Droms, Graph groups, coherence, and three-manifolds, J. Algebra 106 (1987) 484489 MR880971

[13] C Droms, Subgroups of graph groups, J. Algebra 110 (1987) 519-522 MR910401

[14] M Gromov, Groups of polynomial growth and expanding maps, Inst. Hautes Études Sci. Publ. Math. (1981) 53-73 MR623534

[15] J Huang, Quasi-isometry rigidity of right-angled Artin groups, I: The finite out case, preprint (2014) arXiv:1410.8512

[16] M Kapovich, B Kleiner, B Leeb, Quasi-isometries and the de Rham decomposition, Topology 37 (1998) 1193-1211 MR1632904

[17] S-h Kim, T Koberda, Embedability between right-angled Artin groups, Geom. Topol. 17 (2013) 493-530 MR3039768 
[18] S-H Kim, T Koberda, The geometry of the curve graph of a right-angled Artin group, Internat. J. Algebra Comput. 24 (2014) 121-169

[19] T Koberda, Right-angled Artin groups and a generalized isomorphism problem for finitely generated subgroups of mapping class groups, Geom. Funct. Anal. 22 (2012) 1541-1590

[20] L Mosher, M Sageev, K Whyte, Quasi-actions on trees, I: Bounded valence, Ann. of Math. 158 (2003) 115-164 MR1998479

[21] A G Myasnikov, V N Remeslennikov, Exponential groups, II: Extensions of centralizers and tensor completion of CSA-groups, Internat. J. Algebra Comput. 6 (1996) 687-711 MR1421886

[22] P Papasoglu, Quasi-isometry invariance of group splittings, Ann. of Math. 161 (2005) 759-830 MR2153400

Departamento de Matemáticas

Universidad del País Vasco/Euskal Herriko Unibertsitatea

Barrio Sarriena, s/n, 48940 Leioa, Spain

montserrat.casals@ehu .eus

Received: 4 March 2015 Revised: 9 June 2015 